\title{
Feasibility of and patients' perspective on nilotinib dried blood spot self-sampling
}

\author{
Christel C. L. M. Boons ${ }^{1,2}$ (1) $\cdot$ Lonneke Timmers ${ }^{1,2}$. Jeroen J. W. M. Janssen ${ }^{3} \cdot$ Eleonora L. Swart ${ }^{1}$. \\ Jacqueline G. Hugtenburg ${ }^{1,2} \cdot$ N. Harry Hendrikse ${ }^{1,4}$
}

Received: 10 December 2018 / Accepted: 25 January 2019 / Published online: 7 February 2019

(C) The Author(s) 2019

\begin{abstract}
Purpose To obtain insight into the feasibility of, and the patients' perspective on, dried blood spot (DBS) self-sampling by patients with chronic myeloid leukemia (CML) using nilotinib.

Methods Sixty-eight patients with CML using nilotinib participated in this multicenter observational study. Patients were asked to perform blood sampling by means of the DBS method at home just before drug intake (trough level) and to complete a questionnaire including demographics and five questions on their experience with DBS self-sampling.

Results Sixty-one patients $(57.5 \pm 15.0$ years, $49 \%$ female) provided 178 DBS samples of which 137 (77\%) proved useful in clinical practice. Twenty percent of the samples were rejected because the spot size was too small for analysis. A further $3 \%$ were taken at the wrong time. Unsuitable DBS samples were provided by 23 patients. Their educational level was significantly lower than that of patients whose samples were all suitable $(p=0.041)$. Patients considered DBS self-sampling easy and not painful, and three quarters of the patients performed DBS sampling without additional assistance. Patients' belief in the reliability of DBS selfsampling was moderate to high. It was preferred over venous sampling by $37 \%$ of the patients, whereas $39 \%$ had no preference. Conclusion DBS self-sampling by CML patients is feasible in clinical practice provided that patients, particularly those with a lower educational level, are adequately instructed about sample collection with emphasis on timing and volume of sample collection.
\end{abstract}

Keywords Chronic myeloid leukemia $\cdot$ Dried blood spot $\cdot$ Nilotinib $\cdot$ Patients' experiences $\cdot$ Self-sampling

\section{Introduction}

Therapeutic drug monitoring (TDM) of plasma concentrations is becoming increasingly important as a means to personalize the dosing of anticancer drugs [1]. Indications for TDM

Christel C. L. M. Boons

c.boons@vumc.nl

1 Department of Clinical Pharmacology and Pharmacy, Amsterdam UMC, Vrije Universiteit Amsterdam, Cancer Center Amsterdam, Amsterdam, The Netherlands

2 Amsterdam Public Health research institute, Amsterdam UMC, Vrije Universiteit Amsterdam, Amsterdam, The Netherlands

3 Department of Hematology, Amsterdam UMC, Vrije Universiteit Amsterdam, Cancer Center Amsterdam, Amsterdam, The Netherlands

4 Department of Radiology and Nuclear Medicine, Amsterdam UMC, Vrije Universiteit Amsterdam, Cancer Center Amsterdam, Amsterdam, The Netherlands include (the prevention of) side effects, possible drug interactions, suboptimal or delayed responses, treatment failure, and suspected non-adherence $[1,2]$. At present, area under the concentration-time curves (AUC) or trough levels are mostly assayed in serum or plasma obtained by venous blood sampling $[1,3]$. However, venous sampling for AUC measurements requires patients to stay near, or return to, a lab where blood can be drawn, whereas trough level assessment often leads to a delay in drug intake which may complicate adherence to a dosing schedule. Dried blood spot (DBS) sampling is a convenient and validated alternative to venous blood sampling, which increases flexibility in determining blood levels as required for TDM [2]. After adequate training, patients themselves may obtain DBS samples by pricking a finger with an automatic lancet at the required time at home [2, 3]. Samples can be forwarded to the lab before a hospital visit so that results can be discussed at the visit $[3,4]$. These advantages have made DBS self-sampling increasingly popular as a means to monitor a number of oral anticancer drug treatments in daily practice [5-10]. 
However, in spite of being convenient, patients can still be hesitant about self-administering a finger prick [3]. Moreover, DBS obtained by self-sampling may not have the required quality. Mostly, this is the result of difficulties with the size of the blood spot obtained, particularly in the case that the finger prick produced an insufficient amount of blood [3]. Insight into the patient's capability of DBS self-sampling from the patients' perspective is therefore essential before it can be implemented in clinical practice. However, as yet little is known about the feasibility of DBS self-sampling at home $[4,11,12]$.

In chronic myeloid leukemia (CML), a malignant myeloproliferative neoplasm treated with tyrosine kinase inhibitors (TKIs) like imatinib, dasatinib, and nilotinib, TKI plasma concentrations have been related to the efficacy and toxicity of TKI treatment [1, 13-17]. Consequently, the monitoring of TKI plasma concentrations in CML patients has been recommended $[1,15,18,19]$. DBS self-sampling has been shown to be a useful method of TKI blood testing in CML patients and may facilitate the monitoring of TKI plasma concentrations [5, $7,8]$. To support its routine use, we examined the feasibility of, and the patients' perspective on, DBS self-sampling by CML patients using nilotinib.

\section{Methods}

\section{Study design and participants}

The present study data were obtained when performing the RAND study (Response and Adherence to Nilotinib in Daily practice), a multicenter prospective observational study in six Dutch hospitals conducted between August 2013 and April 2017 [20]. Patients ( $\geq 18$ years) with chronic phase CML using nilotinib were eligible for participation. Among other measures, patients were asked to perform blood sampling by means of DBS and to complete a questionnaire.

\section{Blood sampling}

Blood sampling was performed by the patients at home at baseline (if already on treatment) and three time points during nilotinib treatment. Prior to blood sampling, patients received oral instructions by telephone from the investigator (CB) (i.e., warm hands to increase blood flow, prick firmly without hesitation, fill one of four premarked circles on the DBS paper, check fullness of the sampling circle on both sides of the paper). For each sampling time point, patients received a package containing a DBS sampling paper (WhatmanTM, FTATM DMPK-C [GE Healthcare], WB129243, VWR International BV, Amsterdam, Netherlands), a 1.8-mm automatic lancet device (BD Microtainer Contact-Activated Lancet, Pink, BD Diagnostics, Breda, Netherlands), a zip- closure plastic seal bag with a desiccant, a written sampling instruction booklet, and a prepaid envelope.

In order to obtain the trough level $\left(C_{\mathrm{min}}\right)$ at the sampling time point, patients were instructed to take the DBS sample just before taking nilotinib. The time of blood sampling, time of last nilotinib intake and weight were recorded by patients in a diary. $C_{\min }$ samples were defined as those taken between 8 and $16 \mathrm{~h}$ after the prior dose. Patients were instructed to allow the sampling paper to dry for at least $2 \mathrm{~h}$ at room temperature before placing the DBS paper in the seal bag and send it to the laboratory by regular mail service. On arrival, DBS samples were visually inspected on fullness of the sampling circle and presence of a dark-red color on both sides of the paper. The samples were stored at $4{ }^{\circ} \mathrm{C}$ until analysis by means of a previously published nilotinib assay method [5].

\section{Questionnaire}

Patients completed a questionnaire including five questions (self-composed by the investigators) on their experience with DBS self-sampling and questions on demographics. Three items (i.e., "How much pain do you experience from the finger prick" [pain], "How difficult do you find performing blood sampling via the finger prick?" [difficulty], "How reliable do you think blood sampling via the finger prick is?" [reliability]) were scored on a continuous linear scale from zero to 10 . Higher scores indicate more pain, more difficulty, and a stronger belief in the reliability of DBS self-sampling, respectively. Patients were asked whether they needed help to perform blood sampling via the finger prick $(1=$ no, I did it alone, 2 = yes, I needed help). The final question concerned the patient's preference for the method of blood sampling $(1=$ venepuncture (plasma sample), 2 = finger prick (DBS), $3=$ no preference). Demographics included date of birth, gender, level of education, living status, and work status. Level of education was assessed as the highest level completed, and dichotomized into lower education (secondary vocational education or below) and higher education. Living status was categorized as living alone or not living alone and work status as having paid work or not.

\section{Data analyses}

Data were analyzed using SPSS version 22 for Windows (IBM Corp, Armonk, NY, USA). Logistic regression analyses were used to determine differences (in age, gender, education, living alone, work status, patient's perception of pain, difficulty, and reliability of DBS self-sampling, and preference for sampling method) between patients who failed to provide at least one usable DBS sample and patients whose samples were all suitable for analysis. The non-parametric MannWhitney test was used to determine differences in the patients' perception of pain, difficulty, and reliability of DBS sampling 
between the patients who did, and did not, need help performing DBS sampling. A two-sided $p$ value of $\leq 0.05$ was considered statistically significant.

\section{Results}

\section{Patient characteristics}

A total of 68 patients (mean age $57.5 \pm 15.0$ years, $49 \%$ female) were included. Five (7.4\%), two $(2.9 \%)$, and eight (5.9\%) patients dropped out before the first, second, and third time point, respectively. Dropout reasons were treatment discontinuation due to side effects $(n=6)$, disease progression $(n=4)$, entering treatment free remission $(n=4)$, or death $(n=1)$. Patient characteristics are shown in Table 1.

\section{Nilotinib DBS samples}

Sixty-one (90\%) patients provided at least one DBS sample. Seven patients did not provide a sample as the result of study dropout $(n=5)$ and nonresponse $(n=2)$. A total of 178 samples were collected, of which $143(80 \%)$ were suitable for analysis. Of these samples six had been obtained within $8 \mathrm{~h}$ of nilotinib intake (i.e., $0.2-7.8 \mathrm{~h}$ ) and therefore were excluded as $C_{\text {min }}$. In total, 137 (77\%) samples were useful in clinical practice (note, the $C_{\min }$ results are published separately as part of the RAND study).

Thirty-five samples, provided by 23 different patients, were unsuitable because of incomplete $(<5.5-\mathrm{mm})$ filling of the predrawn spot circle. Of these patients, 11 did not successfully take the first DBS sample and 12 patients failed to provide a suitable subsequent sample. As compared to patients whose samples were all suitable, these 23 patients were more often

Table 1 Baseline characteristics $(N=68)$

\begin{tabular}{|c|c|c|}
\hline \multicolumn{2}{|c|}{ Age, range, mean $\pm \mathrm{SD}$ (years) } & $24-87,57.5 \pm 15.0$ \\
\hline \multicolumn{2}{|c|}{ Female gender, $n(\%)$} & $33(48.5 \%)$ \\
\hline \multicolumn{2}{|c|}{ Secondary vocational education or below, $n(\%)$} & $49(72.1 \%)$ \\
\hline \multicolumn{2}{|c|}{ Living alone, $n(\%)$} & $9(13.2 \%)$ \\
\hline \multicolumn{2}{|c|}{ Employed, $n(\%)$} & $30(44.1 \%)$ \\
\hline \multicolumn{2}{|c|}{ Time since $\mathrm{CML}$ diagnosis, range, mean $\pm \mathrm{SD}$ (months) } & $7-268,78 \pm 56^{\mathrm{a}}$ \\
\hline \multicolumn{2}{|c|}{$\begin{array}{l}\text { Duration of nilotinib treatment, range, mean } \pm \text { SD } \\
\text { (months) }\end{array}$} & $3-92,38 \pm 20^{\mathrm{b}}$ \\
\hline \multirow[t]{5}{*}{ Dose, $n(\%)$} & $300 \mathrm{mg}$ BID & $49(72.1 \%)$ \\
\hline & $400 \mathrm{mg}$ BID & $9(13.2 \%)$ \\
\hline & $150 \mathrm{mg}$ BID & $5(7.4 \%)$ \\
\hline & $300 \mathrm{mg}$ QD & $2(2.9 \%)$ \\
\hline & $400 \mathrm{mg}$ QD & $3(4.4 \%)$ \\
\hline
\end{tabular}

$B I D$ twice daily, $Q D$ once daily, $S D$ standard deviation

${ }^{\text {a }}$ Patients not newly diagnosed $(n=48)$

${ }^{\mathrm{b}}$ Patients on nilotinib treatment at study entry $(n=39)$ lower educated ( $87 \%$ vs. $61 \%$, OR $4.2,95 \%$ CI $1.1-17.0, p=$ $0.041)$. No relationship with other characteristics was found.

\section{Patients' perspective on DBS self-sampling}

Fifty-five (90\%) patients completed the questionnaire at the second time point. The median (IQR) scores of the three scale items were $1(0-3), 1(0-5)$, and $7.5(5-8)$ for the perception of pain, difficulty, and reliability, respectively (Table 2). A quarter of the patients $(27 \%)$ needed help in performing DBS sampling. The median score of the difficulty scale for these patients $(4 / 10)$ was higher compared to patients who performed DBS sampling alone (1/10), although this was not statistically significant $(p=0.083)$. With regard to the patients' preference for blood sampling, $37 \%$ of the patients preferred the finger prick (DBS), 24\% preferred venepuncture (plasma sample), and 39\% indicated to have no preference. The number of patients preferring DBS self-sampling over venous sampling was higher in patients whose samples were all suitable (44\% preferring DBS vs. $18 \%$ preferring venepuncture) as compared to patients who failed to provide a usable DBS sample ( $26 \%$ vs. $32 \%$, resp.). This finding, however, was not statistically significant $(p=0.156)$.

\section{Discussion}

The present study aimed to obtain insights into the feasibility of, and patients' perspective on, DBS self-sampling by CML patients using nilotinib. Although the majority of the DBS samples were of clinical value, one fifth of the samples did not fulfill the quality criteria for a reliable analysis of the nilotinib blood level. Interestingly, patients who provided these samples were more often lower educated than patients whose samples were all suitable. A further $3 \%$ of the DBS samples were not useful because they did not represent a trough blood level. Most patients considered DBS self-sampling easy and not painful, and three quarters of the patients performed DBS sampling without additional assistance. Patients' belief in the reliability of DBS self-sampling was moderate to high, and most patients either preferred this method over venous sampling (37\%) or had no preferred method (39\%).

Data on DBS self-sampling are limited to the results of two studies performed in the Netherlands $[4,11]$ and a study performed in Germany [12]. In these studies, the vast majority of the samples (83-88\%) were suitable for analysis. These results are better than those of our study in CML patients, in which only $77 \%$ of the samples were suitable for analysis. However, in all studies including the present study, the main reason for rejecting samples for analysis was the spot size being too small $[4,11$, 12]. Obtaining samples with the right spot size is therefore an important point of action to be addressed before DBS selfsampling can be implemented in clinical practice. In our study, 
Table 2 Patients' perspective on DBS self-sampling $(n=55)$

\begin{tabular}{llr}
\hline & Range & Median (IQR) \\
\hline How much pain do you experience from the finger prick? (0-10) & $0-9$ & $1(0-3)$ \\
How difficult do you find performing blood sampling via the finger prick? (0-10) & $0-10$ & $1(0-5)$ \\
How reliable do you think blood sampling via the finger prick is? (0-10) & $4-10$ & $7.5(5-8)$ \\
Did you need help to perform blood sampling via the finger prick? & \\
No, I did it alone & $73 \%$ \\
Yes, I needed help & $27 \%$ \\
Which type of blood sampling do you prefer? & \\
Venepuncture (plasma sample) & $24 \%$ \\
Finger prick (DBS) & $37 \%$ \\
No preferred method & $39 \%$ \\
\hline
\end{tabular}

$D B S$ dried blood spot, $I Q R$ interquartile range we used an 8-mm spot size for completely filled spots and 5.5$\mathrm{mm}$ standard for smaller spot sizes, as only hole punchers with these sizes were available. Unfortunately, we have been unable to assess the minimal spot size (i.e., quantity of blood) needed for a reliable assay of the nilotinib blood level [5]. Therefore, it may be worthwhile to further explore the correlation between DBS size and plasma/serum TKI concentrations.

The possibility to use small spots, however, may not necessarily result in a higher rate of suitable DBS samples $[4,11$, $12,21]$. The sampling instruction is also an important factor in obtaining suitable DBS samples. In the present study, patients were given oral instructions by telephone as well as a written instruction booklet, whereas in other studies a short training session to practice sample collection was provided $[4,11,12$, 21]. Interestingly, in the present study, the education level of the patients was found to be associated with their ability to deliver a suitable DBS sample as lower educated patients more often delivered samples that were unsuitable for analysis. This suggests that the DBS sampling instruction used in the present study had been inadequate, particularly in patients with a lower education level. Clearly, sampling instructions should be tailored to the educational level of patients while the necessity of completely filling the spot should be emphasized. A training in practice or as a possible alternative, a video instruction, is likely to provide better results.

In the present study, the percentage of patients preferring DBS self-sampling over venous sampling (37\% vs. $24 \%$ ) was lower than in other studies in which $51-61 \%$ of the patients preferred DBS self-sampling over venous sampling (9-11\%) $[4,11,12]$. The lower rate of suitable DBS samples obtained in the present study might relate to this difference as the preference of patients who had difficulty with DBS self-sampling and might have delivered unsuitable samples may have differed from that of patients who had experienced less difficulty with DBS self-sampling. Indeed, the preference for DBS selfsampling over venous sampling was somewhat higher in patients whose samples were all suitable as compared to patients who failed to provide a usable DBS sample.
A limitation of this study is the DBS self-sampling instruction which may have been inadequate, particularly in patients with a lower education level. This appears to have influenced the number of suitable samples delivered and the preference of patients for blood sampling methods. Selection bias may have occurred, because the present study data were obtained when performing the RAND study [20]. Patients' perspectives on DBS self-sampling might have been affected by their participation in the RAND study.

In conclusion, DBS self-sampling by CML patients appears feasible in clinical practice provided that patients, particularly those with a lower education level, are adequately instructed about sample collection. Sampling instructions should be clear and straightforward, emphasize timing and volume of sample collection, and preferably completed with training in practice. In order to increase DBS sample suitability, it is worthwhile to explore the usefulness of smaller spot sizes.

Acknowledgements The data collection for this work was supported by an unrestricted grant by Novartis. The authors are responsible for the content of the article. We thank the patients and all study personnel in the different centers for their efforts. We thank research analyst René Vos for the sample analysis and Jan Jacob Beckeringh for his editorial assistance.

Author contributions $\mathrm{CB}, \mathrm{LT}, \mathrm{JJ}, \mathrm{ES}$, and JH were involved in conception and design of the study. CB was involved in acquisition and analysis of the data. CB drafted the manuscript and LT, JJ, ES, JH, and $\mathrm{NH}$ critically revised the manuscript. All authors gave the final approval of the manuscript to be published. ES was the principal investigator.

Funding This study was funded by Novartis (grant number CAMN107ENL04T).

\section{Compliance with ethical standards}

Conflict of interest J.J.W.M. Janssen is an advisory board member for Abbvie, Incyte, Jazz Pharmaceuticals, and Pfizer and has received research support funding from Novartis and Bristol Myers Squibb and speaker's fees from Incyte and Pfizer. The other authors declare no conflict of interest. 
Ethical approval The study was approved by the Medical Ethics review board of Amsterdam University Medical Centers (location VUmc, Amsterdam) (2013.035), as well as the ethical boards of each participating center. All procedures performed were in accordance with the ethical standards of the Medical Ethics review board and with the 1964 Helsinki declaration and its later amendments or comparable ethical standards. Informed consent was obtained from all individual participants included in the study.

Open Access This article is distributed under the terms of the Creative Commons Attribution 4.0 International License (http:// creativecommons.org/licenses/by/4.0/), which permits unrestricted use, distribution, and reproduction in any medium, provided you give appropriate credit to the original author(s) and the source, provide a link to the Creative Commons license, and indicate if changes were made.

\section{References}

1. Verheijen RB, Yu H, Schellens JHM, Beijnen JH, Steeghs N, Huitema ADR (2017) Practical recommendations for therapeutic drug monitoring of kinase inhibitors in oncology. Clin Pharmacol Ther 102(5):765-776. https://doi.org/10.1002/cpt.787

2. Wilhelm AJ, den Burger JC, Swart EL (2014) Therapeutic drug monitoring by dried blood spot: progress to date and future directions. Clin Pharmacokinet 53(11):961-973. https://doi.org/10. 1007/s40262-014-0177-7

3. Edelbroek PM, van der Heijden J, Stolk LML (2009) Dried blood spot methods in therapeutic drug monitoring: methods, assays, and pitfalls. Ther Drug Monit 31(3):327-336

4. Jager NG, Rosing H, Linn SC, Schellens JH, Beijnen JH (2015) Dried blood spot self-sampling at home for the individualization of tamoxifen treatment: a feasibility study. Ther Drug Monit 37(6): 833-836. https://doi.org/10.1097/FTD.0000000000000224

5. Boons CC, Chahbouni A, Schimmel AM, Wilhelm AJ, den Hartog YM, Janssen JJ, Hendrikse NH, Hugtenburg JG, Swart EL (2017) Dried blood spot sampling of nilotinib in patients with chronic myeloid leukaemia: a comparison with venous blood sampling. J Pharm Pharmacol 69(10):1265-1274. https://doi.org/10.1111/jphp. 12757

6. Nijenhuis CM, Huitema AD, Marchetti S, Blank C, Haanen JB, van Thienen JV, Rosing H, Schellens JH, Beijnen JH (2016) The use of dried blood spots for pharmacokinetic monitoring of vemurafenib treatment in melanoma patients. J Clin Pharmacol. https://doi.org/ $10.1002 /$ jcph. 728

7. Kralj E, Trontelj J, Pajic T, Kristl A (2012) Simultaneous measurement of imatinib, nilotinib and dasatinib in dried blood spot by ultra high performance liquid chromatography tandem mass spectrometry. J Chromatogr B Analyt Technol Biomed Life Sci 903:150-156

8. Antunes MV, Raymundo S, Wagner SC, Mattevi VS, Vieira N, Leite R, Reginato F, Capra MZ, Fogliatto L, Linden R (2015) DBS sampling in imatinib therapeutic drug monitoring: from method development to clinical application. Bioanalysis 7(16):21052117. https://doi.org/10.4155/bio.15.101

9. de Wit D, den Hartigh J, Gelderblom H, Qian Y, den Hollander M, Verheul H, Guchelaar HJ, van Erp NP (2015) Dried blood spot analysis for therapeutic drug monitoring of pazopanib. J Clin Pharmacol 55(12):1344-1350. https://doi.org/10.1002/jcph.558
10. Willemsen A, Knapen LM, de Beer YM, Bruggemann RJM, Croes S, van Herpen CML, van Erp NP (2017) Clinical validation study of dried blood spot for determining everolimus concentration in patients with cancer. Eur J Clin Pharmacol 74:465-471. https:// doi.org/10.1007/s00228-017-2394-0

11. Kromdijk W, Mulder JW, Smit PM, Ter Heine R, Beijnen JH, Huitema AD (2013) Therapeutic drug monitoring of antiretroviral drugs at home using dried blood spots: a proof-of-concept study. Antivir Ther 18(6):821-825. https://doi.org/10.3851/IMP2501

12. Leichtle AB, Ceglarek U, Witzigmann H, Gabel G, Thiery J, Fiedler GM (2010) Potential of dried blood self-sampling for cyclosporine c(2) monitoring in transplant outpatients. J Transp Secur 2010:201918. https://doi.org/10.1155/2010/201918

13. Larson RA, Yin OQ, Hochhaus A, Saglio G, Clark RE, Nakamae H, Gallagher NJ, Demirhan E, Hughes TP, Kantarjian HM, le Coutre PD (2012) Population pharmacokinetic and exposureresponse analysis of nilotinib in patients with newly diagnosed $\mathrm{Ph}+$ chronic myeloid leukemia in chronic phase. Eur J Clin Pharmacol 68(5):723-733

14. Giles FJ, Yin OQ, Sallas WM, le Coutre PD, Woodman RC, Ottmann OG, Baccarani M, Kantarjian HM (2013) Nilotinib population pharmacokinetics and exposure-response analysis in patients with imatinib-resistant or -intolerant chronic myeloid leukemia. Eur J Clin Pharmacol 69(4):813-823

15. Miura M (2015) Therapeutic drug monitoring of imatinib, nilotinib, and dasatinib for patients with chronic myeloid leukemia. Biol Pharm Bull 38(5):645-654. https://doi.org/10.1248/bpb.b15-00103

16. Picard S, Titier K, Etienne G, Teilhet E, Ducint D, Bernard MA, Lassalle R, Marit G, Reiffers J, Begaud B, Moore N, Molimard M, Mahon FX (2007) Trough imatinib plasma levels are associated with both cytogenetic and molecular responses to standard-dose imatinib in chronic myeloid leukemia. Blood 109(8):3496-3499. https://doi.org/10.1182/blood-2006-07-036012

17. Guilhot F, Hughes TP, Cortes J, Druker BJ, Baccarani M, Gathmann I, Hayes M, Granvil C, Wang Y (2012) Plasma exposure of imatinib and its correlation with clinical response in the tyrosine kinase inhibitor optimization and selectivity trial. Haematologica 97(5):731-738. https://doi.org/10.3324/haematol.2011.045666

18. Hochhaus A, Saussele S, Rosti G, Mahon FX, Janssen J, HjorthHansen H, Richter J, Buske C, Committee EG (2017) Chronic myeloid leukaemia: ESMO Clinical Practice Guidelines for diagnosis, treatment and follow-up. Ann Oncol 28(suppl 4):iv41-iv51. https://doi.org/10.1093/annonc/mdx219

19. Jabbour E, Kantarjian H (2018) Chronic myeloid leukemia: 2018 update on diagnosis, therapy and monitoring. Am J Hematol 93(3): 442-459. https://doi.org/10.1002/ajh.25011

20. Boons CC, Swart EL, Timmers L, van de Ven PM, Janssen JJ, Hugtenburg JG (2014) Study protocol of the RAND-study: a multicenter, prospective cohort study investigating response and adherence to nilotinib treatment in chronic myeloid leukemia. BMC Cancer 14:247

21. Cheung CY, van der Heijden J, Hoogtanders K, Christiaans M, Liu YL, Chan YH, Choi KS, van de Plas A, Shek CC, Chau KF, Li CS, van Hooff J, Stolk L (2008) Dried blood spot measurement: application in tacrolimus monitoring using limited sampling strategy and abbreviated AUC estimation. Transpl Int 21(2):140-145. https:// doi.org/10.1111/j.1432-2277.2007.00584.x

Publisher's note Springer Nature remains neutral with regard to jurisdictional claims in published maps and institutional affiliations. 